\title{
Topological Lifshitz transition of the intersurface Fermi-arc loop in $\mathrm{NbIrTe}_{4}$
}

\author{
S. A. Ekahana $\odot,{ }^{1,2,}{ }^{*}$ Y. W. Li, ${ }^{1,}{ }^{*}$ Y. Sun, ${ }^{3}$ H. Namiki $\odot,{ }^{4}$ H. F. Yang, ${ }^{5}$ J. Jiang, ${ }^{5,6}$ L. X. Yang, ${ }^{7}$ W. J. Shi $\odot,{ }^{3}$ C. F. Zhang, ${ }^{1,8}$ \\ D. Pei, ${ }^{1}$ C. Chen $\odot,{ }^{1,5,9}$ T. Sasagawa, ${ }^{4}$ C. Felser, ${ }^{3,10,11}$ B. H. Yan, ${ }^{12}$ Z. K. Liu, ${ }^{5, \dagger}$ and Y. L. Chen ${ }^{1,5,7,9, \frac{1}{,}}$ \\ ${ }^{1}$ Department of Physics, University of Oxford, Oxford, OX1 3PU, United Kingdom \\ ${ }^{2}$ Swiss Light Source, Paul Scherrer Institute, CH-5232 Villigen, Switzerland \\ ${ }^{3}$ Max Planck Institute for Chemical Physics of Solids, D-01187 Dresden, Germany \\ ${ }^{4}$ Materials and Structures Laboratory, Tokyo Institute of Technology, Yokohama, Kanagawa 226-8503, Japan \\ ${ }^{5}$ School of Physical Science and Technology, ShanghaiTech University and CAS-Shanghai Science Research Center, \\ Shanghai 200031, People's Republic of China \\ ${ }^{6}$ Advanced Light Source, Lawrence Berkeley National Laboratory, Berkeley, California 94720, USA \\ ${ }^{7}$ State Key Laboratory of Low Dimensional Quantum Physics, Department of Physics and Collaborative Innovation Center \\ of Quantum Matter, Tsinghua University, Beijing 100084, People's Republic of China \\ ${ }^{8}$ College of Advanced Interdisciplinary Studies and Interdisciplinary Center for Quantum Information, \\ National University of Defense Technology, Changsha 410073, People's Republic of China \\ ${ }^{9}$ ShanghaiTech Laboratory for Topological Physics, Shanghai 200031, People's Republic of China \\ ${ }^{10}$ John A. Paulson School of Engineering and Applied Sciences, Harvard University, Cambridge, Massachusetts 02138, USA \\ ${ }^{11}$ Department of Physics, Harvard University, Cambridge, Massachusetts 02138, USA \\ ${ }^{12}$ Weizmann Institute of Science, Herzl St 234, Rehovot, 76100, Israel
}

(Received 9 October 2019; accepted 28 July 2020; published 13 August 2020)

\begin{abstract}
Surface arcs (SAs) or Fermi arcs connecting pairs of bulk Weyl points with opposite chiralities are the signatures of Weyl semimetals in angle-resolved photoemission spectroscopy (ARPES) studies. The nontrivial topology of the bulk band structure guarantees the existence of these exotic Fermi arcs with connectivity that is strongly dependent on the surface. It has been theoretically proposed and experimentally confirmed that Fermi arcs at opposite surfaces can complete an unusual closed cyclotron orbit called a Weyl orbit, which leads to various intriguing transport properties. In this paper, a systematic ARPES study on opposite terminations (001) of type-II Weyl semimetal $\mathrm{NbIrTe}_{4}$ reveals different Fermi arc connections which result in a unique closed intersurface Fermi arc loop configurations (combining both projections of SAs) containing two pairs of Weyl points. In particular, the top surface ARPES data and corresponding ab initio calculation suggests that a topological Lifshitz transition occurs by tuning the chemical potential. SA rewiring on the top surface opens the intersurface arc loop at the Weyl node energy level into an open line, challenging the close-orbit description and leading to an unexplored scenario. Our results demonstrate the intrinsic alteration of Fermi arc connections and propose $\mathrm{NbIrTe}_{4}$ as a potential platform to examine Fermi-arc related phenomenon.
\end{abstract}

DOI: 10.1103/PhysRevB.102.085126

\section{INTRODUCTION}

\section{A. Topological Weyl semimetal}

The fascinating physical properties of topological Weyl semimetals (TWSs) originate from their unusual bulk electronic structure relativistic chiral Weyl nodes as well as the nontrivial surface-state Fermi arcs connecting each pair of Weyl nodes [1-4]. Moreover, the TWS has a family called type-II TWSs whose Weyl nodes are highly anisotropic and tilted, breaking the Lorentz symmetry [5-7]. The nonsaturated extremely large magnetoresistance discovered in these type-II

\footnotetext{
*These authors contributed equally to this work.

†liuzhk@shanghaitech.edu.cn

${ }^{\ddagger}$ yulin.chen@physics.ox.ac.uk
}

TWSs, such as $\mathrm{MoTe}_{2}$ and $\mathrm{TaIrTe}_{4}$, is probably due to a synergetic effect of electron-hole compensation, spin-orbit interaction, and ultrahigh carrier mobility [8-10].

Fermi arcs, on the other hand, are usually viewed as important fingerprints of the nontrivial topology of TWSs since there are no analogs in a conventional two-dimensional system $[11,12]$. However, it has long been a puzzle whether these exotic surface states can contribute to any novel macroscopic properties. Without a global bulk gap, the transport behaviors of TWSs are usually dominated by bulk electrons. Furthermore, by coupling with bulk states, theorists point out the Fermi arc transport is dissipative [13] in contrast to the ballistic conductance of Dirac fermions on the surface of topological insulators [14]. This picture has greatly changed by recent theoretical proposals [15-17] and experimental observations [18] of Weyl orbits, which provides the key ingredient for Fermi-arc-related potential applications [19-22]. 

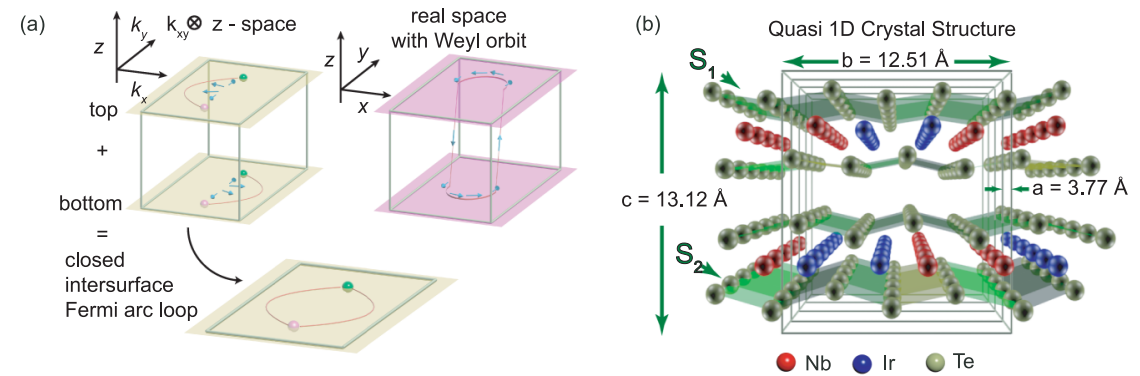

(c)

Core Level, 3D XRD, \& Sample

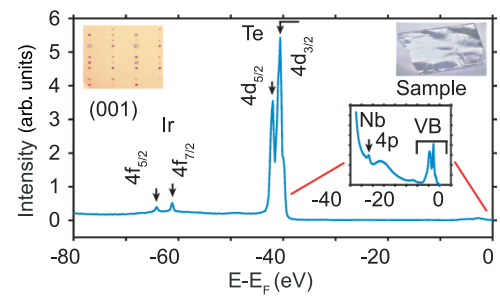

(d)

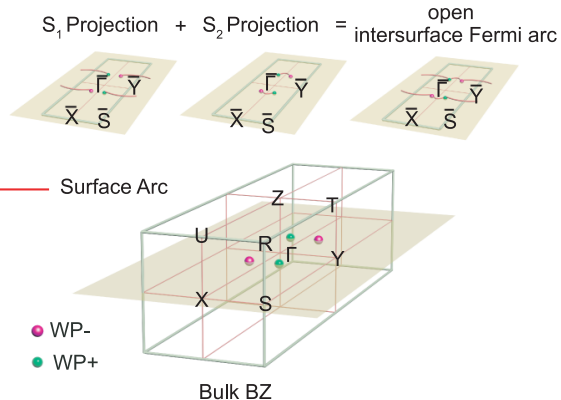

(e) Berry curvature calculation $\left(k_{z}=0\right)$

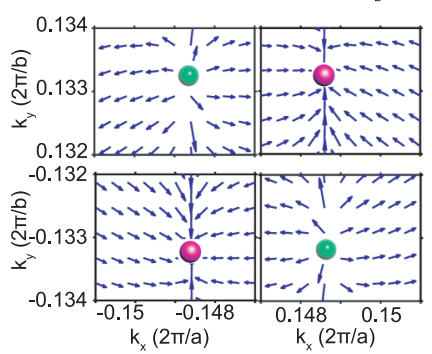

(f) Experimental and calculated bulk band comparison
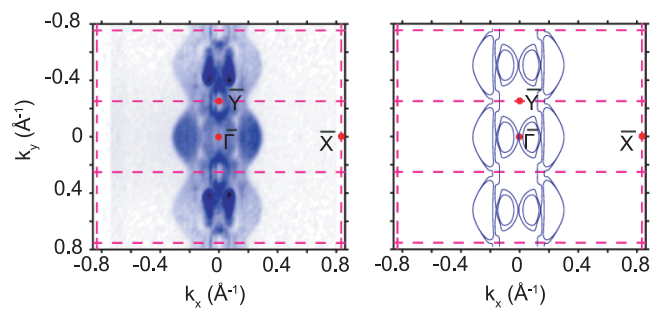

FIG. 1. (a) Weyl orbit created from the top and bottom Fermi arcs tunneling through the bulk as shown in momentum space and real space. The closed intersurface Fermi arc loop is shown, which is the combined shape of both arcs from opposite surfaces. (b) Crystal structure of $\mathrm{NbIrTe}_{4}$ shows a quasi-1D feature along the $a$ direction. Crystal lattice constants are labeled. $S_{1}$ and $S_{2}$ indicate the top and bottom surfaces of each unit cell. They are inequivalent due to the glide mirror operation $\left\{m_{010} \mid \frac{1}{2}, 0, \frac{1}{2}\right\}$. (c) Core level, x-ray diffraction patterns, and photos of sample cleavage system. (d) Schematic of the proposed Weyl point (WP) locations and Fermi arcs in the projected BZ of $S_{1}$ and $S_{2}$ surface (upper panel) of $\mathrm{NbIrTe}_{4}$ as well as the WP locations in the bulk 3D BZ (lower panel). The open intersurface Fermi arc also shown differs from the closed version in (a). (e) Berry curvature calculation around the proposed WP locations. (f) ARPES data on the broad Fermi surface map of $\mathrm{NbIrTe}_{4}$ (taken from $S_{1}$ surface) across three BZs agree well with the calculated bulk band.

\section{B. Weyl orbit}

Differing from conventional cyclotron orbits transferring quasiparticle momentum by Lorentz force, a Weyl orbit conveys chirality among Weyl nodes [Fig. 1(a)] [15,16,18]. On the surface, surface arcs (SAs) act as Fermi-level plumbing conduits conducting quasiparticles from one Weyl node to another [23]. The wormhole intersurface transfer between two Weyl nodes at opposite surfaces is realized through the chiral bulk states under zero Lorentz force $[15,16,18]$. Based on this building block combining both SAs and topological chiral bulk states, more exotic effects are proposed, including 3D quantum Hall effect, nonlocal voltage generation, and resonances in the transmission of electromagnetic waves [19-22,24]. Thus far, Fermi-arc-mediated chirality transfer has been realized in Dirac semimetal $\mathrm{Cd}_{3} \mathrm{As}_{2}$ in which the Weyl orbit connects the same pair of Weyl nodes from opposite surfaces and thus create a closed intersurface Fermi arc loop [Fig. 1(a)] [18].

In this paper, by using angle-resolved photoemission spectroscopy (ARPES) combined with ab initio calculations, we investigate a candidate for type-II TWS, $\mathrm{NbIrTe}_{4}$, and observe clear SAs that suggest a closed intersurface Fermi arc loop configuration which connects two different pairs of Weyl nodes from the top and bottom surfaces in the type-II TWS candidate $\mathrm{NbIrTe}_{4}$. Furthermore, calculations on the top surface further show a topological Lifshitz transition when the chemical potential is shifted to the Weyl points where the new
SAs now connect a different pair of Weyl points (WPs) than before, creating an open intersurface Fermi arc configuration [Fig. 1(d)]. This finding poses a challenge on the closedorbit description of Fermi arc configuration if we consider both top and bottom surfaces together, opening an unexplored situation.

\section{SAMPLE CHARACTERIZATION AND AB INITIO CALCULATION}

$\mathrm{NbIrTe}_{4}$ has a noncentrosymmetric crystal structure as shown in Fig. 1(b) that has space group Pmn21 with $a=3.77 \pm 0.01 \AA, b=12.51 \pm 0.01 \AA$, and $c=13.12 \pm$ $0.01 \AA$, according to our x-ray diffraction (XRD) measurements and in good agreement with the previous work in Ref. [25] [the inset of Fig. 1(c) for the XRD pattern of the (001) surface and core level in Fig. 1(c) confirms the elemental composition]; it can be viewed as the doubled $\mathrm{WTe}_{2}$ and $\mathrm{MoTe}_{2}$ in $T_{d}$ phase (holding two Te-(Nd,Ir)-Te layers within one unit cell) where we have Peierls distortion along $b$ direction on the Te atom sheet. We notice the top layer is in glide symmetry (mirrored on $x z$ plane and translated) with respect to the bottom layer. Therefore, it creates two types of exposed surfaces after cleaving named as $S_{1}$ (top surface) or $S_{2}$ (bottom surface) as displayed in Fig. 1(b) on which we performed ARPES measurements [Fig. 1(f) for general ARPES result]. 

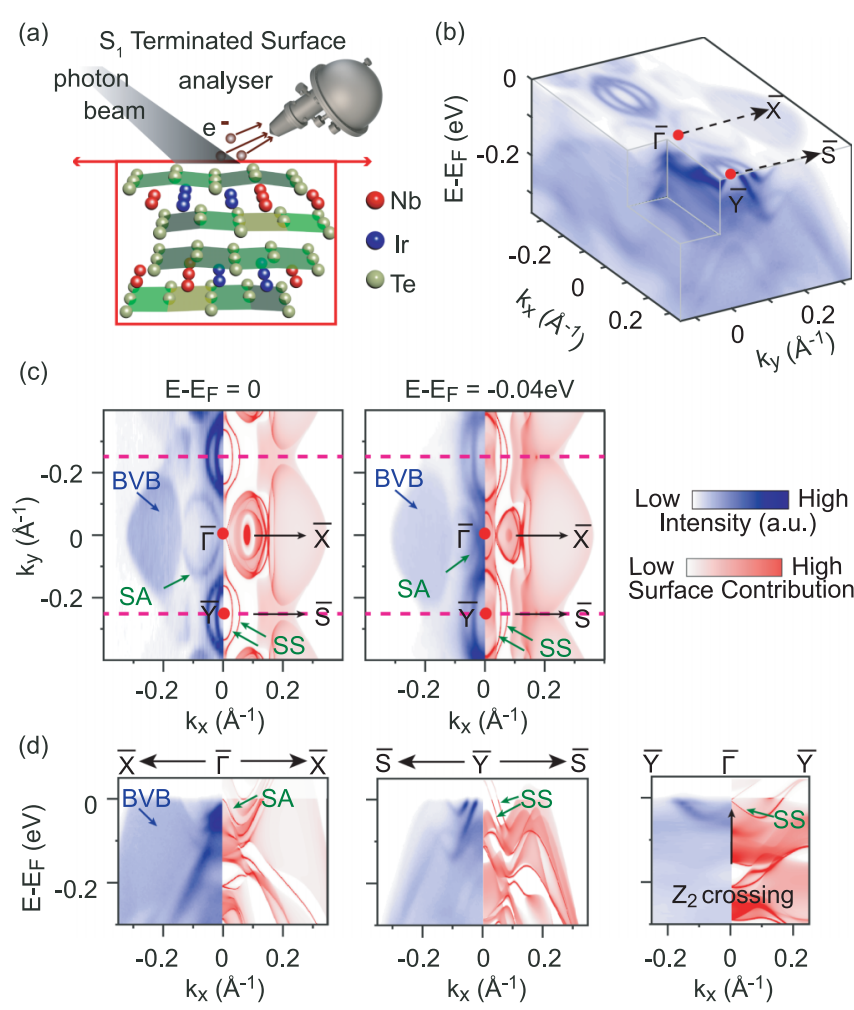

FIG. 2. (a) The schematic of ARPES measurement on the $S_{1}$ termination. (b) Three-dimensional (3D) ARPES data of $\mathrm{NbIrTe}_{4}$ on the $S_{1}$ termination. (c) Measured (left side) and calculated (right side) Fermi surface (left panel) and energy contour at $40 \mathrm{meV}$ below $E_{F}$ (right panel) of $S_{1}$ termination with the surface arcs (SAs), surface states (SSs), and the bulk valence band (BVB) labeled. (d) Photoemission intensity (left side) and calculation results (right side) along the high symmetry $\overline{\mathrm{X}} \bar{\Gamma} \overline{\mathrm{X}}, \overline{\mathrm{S}} \overline{\mathrm{Y}} \overline{\mathrm{S}}$, and $\overline{\mathrm{Y}} \bar{\Gamma} \overline{\mathrm{Y}}$ directions with the dispersion of the SA, SS, and the BVB features labeled. In $\bar{Y} \bar{\Gamma} \bar{Y}$ cut, the crossing in the SS with a $Z_{2}$ invariant is labeled.

Due to the absence of inversion symmetry, $\mathrm{NbIrTe}_{4}$ is proposed to host four type-II WPs (see Supplemental Material Fig. SI.1b [26]) pinned on $k_{z}^{\prime}=0$ (due to the joint glide $c_{2 z}$ and time-reversal symmetry) [6,27] and $\left(k_{x}^{\prime}\right.$, $\left.k_{y}^{\prime}\right)=\left( \pm 0.1486 \frac{2 \pi}{a}, \pm 0.13333 \frac{2 \pi}{b}\right)=( \pm 0.248, \pm 0.067) \AA^{-1}$ [Figs. 1(c) and 1(d)], the minimum number of WPs in an inversion breaking WSM system; contradicting the prediction of 16 WPs in Ref. [28]. The Berry curvature calculation further confirm that only four WPs exist as they behave as sources and sinks only around these four points (Fig. 1(e) and Supplemental Material A.1. [29]). Our calculation also shows that the WP comes from the intersection of tilted cone (type-II Weyl node) crossing above the Fermi energy at $E-E_{F}=0.131 \mathrm{eV}$ (see Supplemental Material Fig. SI.1 [30]).

Prominently, previous calculations (for both $\mathrm{NbIrTe}_{4}$ and $\mathrm{TaIrTe}_{4}$ ) have suggested the different shape and connectivity of SA on the two different surfaces: SA on $S_{1}$ surface connects the WPs along the $k_{y}$ direction to the neighboring Brillouin zone (BZ) while on $S_{2}$ surface, it connects WPs along $k_{y}$ direction within the same BZ, creating an open intersurface orbit as shown in Fig. 1(d) [27,31]. Previous works in laser (a)

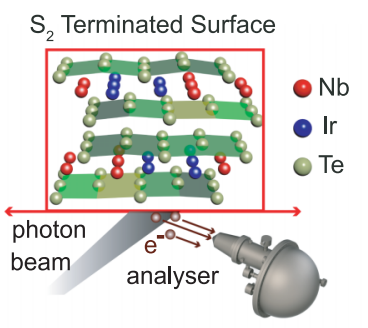

(b)
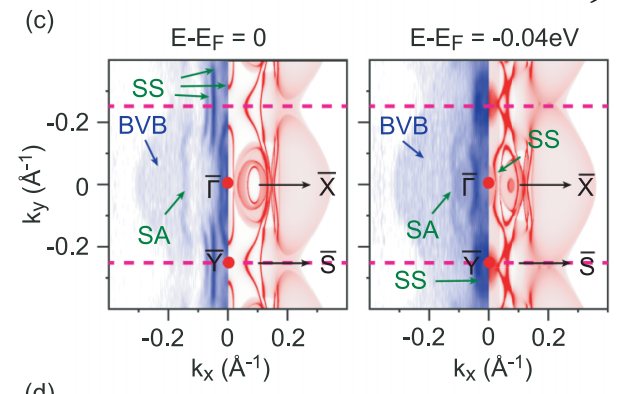

Low High

Intensity (arb. units)

Low High
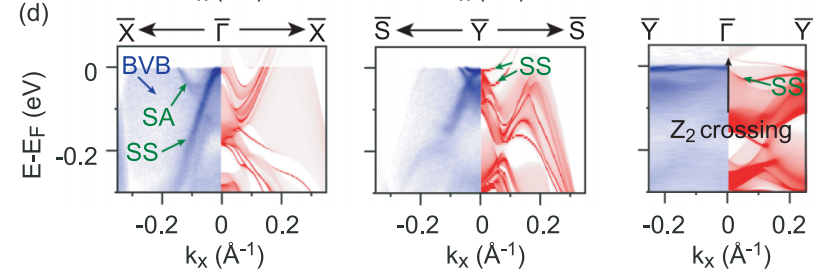

FIG. 3. (a) The schematic of ARPES measurement on the $S_{2}$ termination. (b) Three-dimensional (3D) ARPES data of $\mathrm{NbIrTe}_{4}$ on the $S_{2}$ termination. (c) Measured (left side) and calculated (right side) Fermi surface (left panel) and energy contour at $40 \mathrm{meV}$ below $E_{F}$ (right panel) of $S_{2}$ termination with the surface arcs (SAs), surface states (SSs), and the bulk valence band (BVB) labeled. (d) Photoemission intensity (left side) and calculation results (right side) along the high symmetry $\bar{X} \bar{\Gamma} \bar{X}, \bar{S} \bar{Y} \bar{S}$, and $\bar{Y} \bar{\Gamma} \bar{Y}$ directions with the dispersion of the SA, SS, and the BVB features labeled. In $\bar{Y} \bar{\Gamma} \bar{Y}$ cut, the crossing in the SS with a $Z_{2}$ invariant is labeled.

ARPES [32] and spin-resolved ARPES [33] have attempted to reveal this unique arc configuration in $\mathrm{TaIrTe}_{4}$ and yet the detailed Fermi surface on each terminations remain unclear. In this paper, we present detailed ARPES measurements from $\mathrm{NbIrTe}_{4}$ that shed a different configuration as demonstrated below.

\section{ARPES RESULTS}

\section{A. ARPES results on top surface}

Figure 2 demonstrates the high-resolution ARPES data on the $S_{1}$ termination [see Fig. 2(a) for the illustration of the measurement configuration]. From the 3D plot of the electronic band structure [Fig. 2(b)], as well as the Fermi surface map [Fig. 2(c), left panel], we could identify several key features, including a distinctive hourglass-shaped feature around $\bar{\Gamma}$, an eye-shaped feature around $\overline{\mathrm{Y}}$, as well as a blurred pocket on the Fermi surface. By comparing with the ab initio calculations [right side of Fig. 2(c), see Supplemental Material A.1. and A.2. [34]), we can see that the hourglass and the eye shapes have the strongest surface contribution while the blurred pocket shows its bulk origin. Furthermore, 


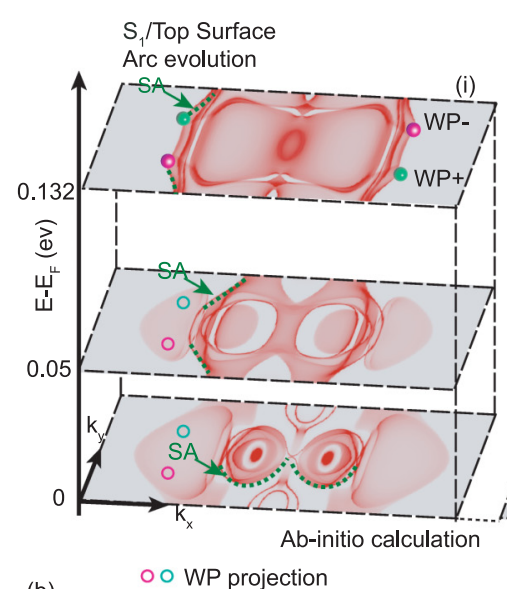

(b)

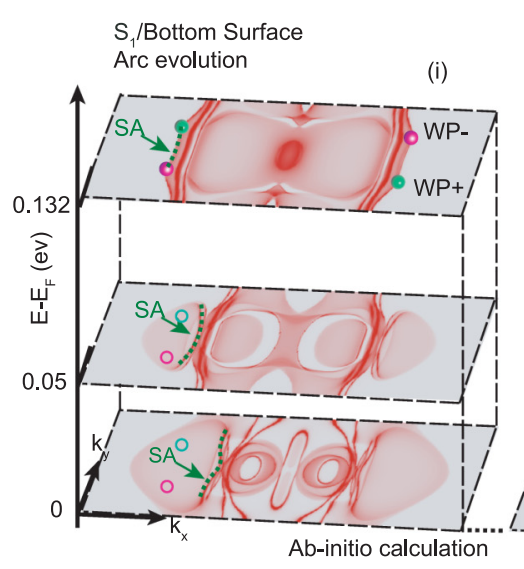

(ii)
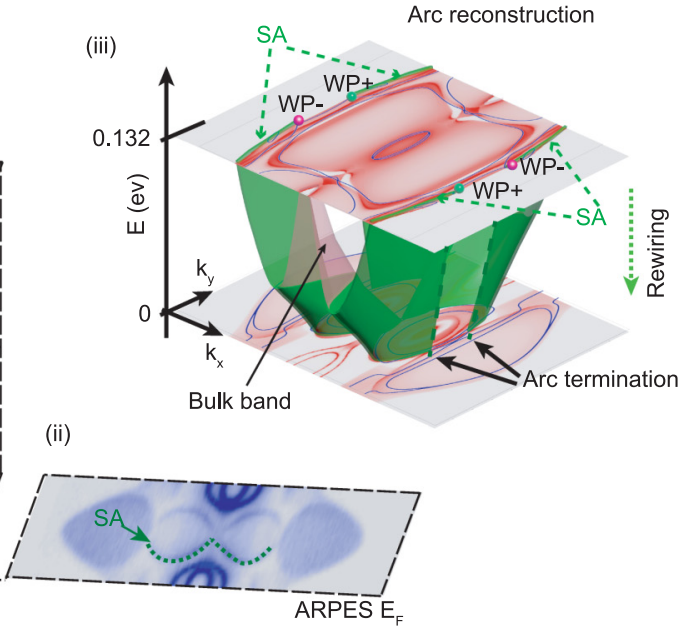

(iii)

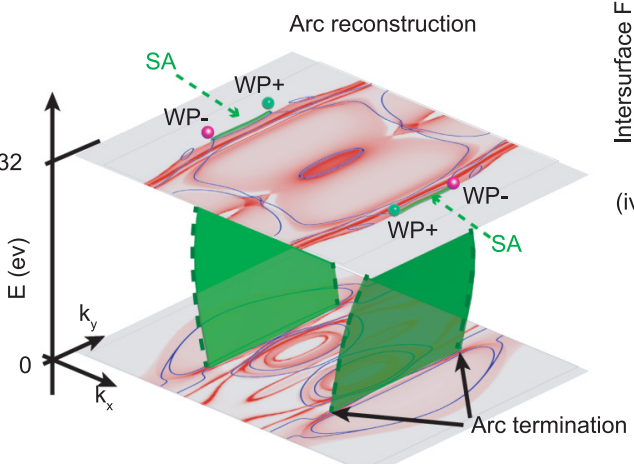

0.132

(ii)

(iv)

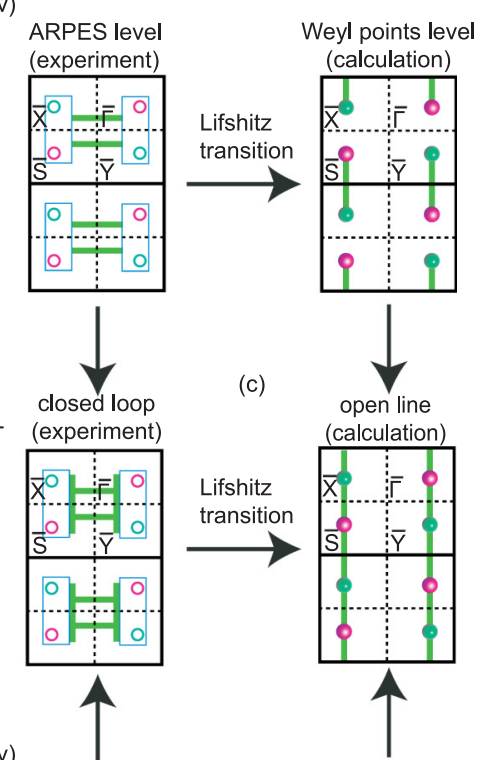

$$
\begin{aligned}
& \text { AR } \\
& \hline \text { ex } \\
& \hline \\
& \hline
\end{aligned}
$$

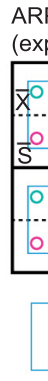

ARPES level (experiment)

Weyl points level

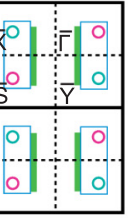
(calculation)

no transition

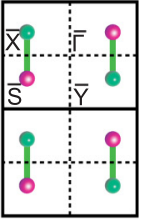

hole pocket at ARPES position

FIG. 4. (a)(i), (b)(i) Calculated data from Weyl point energy position, an intermediate energy level, and ARPES Fermi level are shown. (a)(ii), (b)(ii) ARPES Fermi surface result $\left[S_{1}\right.$ (a) and $S_{2}$ (b)] is shown to demonstrate the agreement with the calculated result in panel (i). Panel (iii) describes the SA evolution from the WPs position at $(E=0.132 \mathrm{eV})$ to the Fermi energy level of the ARPES measurement in 3D reconstruction. The $S_{1}$ surface shows a rewiring of SAs at different binding energies, which is attributed to the hybridization between surface (green surface) and bulk band (pink surface). The constant energy contour at $0.132 \mathrm{eV}$ above $E_{F}$ and at $E_{F}$ shows the overlapped plot of the DFT calculation (blue solid lines, where the Weyl fermion and WPs are clearly seen) and surface slab calculations (red intensities, where the bulk continuum and the Fermi arcs are clearly seen). Panel (iv) describe the Fermi arc connection for both $S_{1}$ and $S_{2}$ surfaces with panel (c) as the combination of both projections creating the intersurface Fermi-arc loop. It can be seen the ARPES position shows a closed loop while at the Weyl points position the loop becomes an open line.

the eye shape and the blurred pocket increases their sizes at higher binding energy while the hourglass-shaped feature reduces its size [Fig. 2(c), right panel], showing they are hole and electron pocket(s), respectively. Prominently, we notice that the hourglass shape is not a closed contour where the start and the end are near the projection of the bulk WPs. We attribute this shape to the SA band that connects pairs of WPs $\left( \pm k_{x}^{\prime}, k_{y}^{\prime}\right)$, which lies above the Fermi Energy $\left(E_{F}\right)$ according to the analysis of trivial/nontrivial $Z_{2}$ invariants along high symmetry cuts (see Supplemental Materials A.1. for detailed explanation $[29,36])$. It should be noted that the arc connection differs from the arc connection at the WP position as displayed before in Fig. 1(d), which will be discussed later. Meanwhile, the eye shapes around $\bar{Y}$ form a closed contour where the inner eye shape [see $\bar{Y} \bar{\Gamma} \bar{Y}$ cut at Fig. 2(d), right panel] shows that it forms a crossing at the high symmetry point of $\bar{\Gamma}$ confirmed by the calculation, which in turn also forms the hourglass-shaped band. The linear crossing at the $\bar{\Gamma}$ point is protected, stemming from the band inversion found in bulk calculations and confirmed to have a nonzero $Z_{2}$ invariant.

\section{B. ARPES results on bottom surface}

The ARPES measurement on the $S_{2}$ surface of $\mathrm{NbIrTe}_{4}$ [Fig. 3(a)] shows relevant but different electronic structures compared with the $S_{1}$ surface [see Fig. 3(b) for the 3D plot of the electronic structure, Fig. 3(c) for the constant energy contours and Fig. 3(d) for the high symmetry cuts]. The blurred bulk feature remains similar, confirming its bulk origin thus not sensitive to the condition of termination. However, the SS and SA bands are significantly different as compared with the $S_{1}$ surface: The SA now becomes straight lines with finite lengths on the Fermi level and connects the projection 
of another pair of WPs $\left(k_{x}^{\prime}, \pm k_{y}^{\prime}\right)$, which shows different connectivity compared to the $S_{1}$ surface [Fig. 2(b)], yet consistent connectivity with Fig. 1(d) and agreeing with previous works $[32,33]$. The SS also deviates from the previous eye shape and becomes a curved line going parallel along the $\bar{\Gamma} \bar{Y}$ direction. Due to the hybridization with the bulk band, the intensity of SS becomes weaker close to $\bar{\Gamma}$ at $E_{F}$. Lastly, the high symmetry $\bar{Y} \bar{\Gamma} \bar{Y}$ cut shows that the elliptical band observed near the $\mathrm{BZ}$ center evolves into the $Z_{2}$ invariant crossing above the Fermi level similar to the $S_{1}$ surface before.

\section{LIFSHITZ TRANSITION IN NbIrTe}

Here we discuss the SA assignment for the $S_{1}$ surface at the ARPES Fermi level as it differs from the SA assignment at the WP energy position $\left(\sim 132 \mathrm{meV}\right.$ above $\left.E_{F}\right)$, while the $S_{2}$ surface SAs do not change connectivity; SA itself is still well defined away from the WP energy level before merging completely into bulk at different energy [23]. Figures 4(i) and 4(ii) for both $S_{1}$ (a) and $S_{2}$ (b) surfaces show a clear agreement between the ARPES and calculations. As we follow the evolution of the Fermi surface map toward the WPs energy (in calculation), we notice that a Lifshitz transition occurs at the $S_{1}$ surface (rewiring of Fermi arc); from connecting WPs along $k_{y}$ at different $\mathrm{BZs}$ to connecting WPs along $k_{x}$ within the same BZ [schematic shown in Figs. 4(iii) and 4(iv)]. We attribute this finding due to the hybridization that happens between the surface states and the bulk states that are in close proximity; while the SA itself is able to rewire due to perturbation $[37,38]$. To further visualize the evolution of the SA at different binding energies, Figs. 4(a)(iii) and 4(b)(iii) demonstrate the evolution of the energy band (SA band with green surface and the bulk band with pink surface) from WP position toward the ARPES Fermi level position. We can see that the SA for the $S_{2}$ evolves smoothly, maintaining the original connection while the SA at $S_{1}$ changes connectivity as it follows the evolution of the bulk band shown with pink surface. As a consequence, we also notice that the intersurface Fermi-arc loop created by these two surfaces change shape, creating this Lifshitz transition: closed orbit at the ARPES position and open line at WP position [Fig. 4(c)]. Despite the rewiring, the projected SA from both surfaces still connect two oppositely charged WPs and thus is consistent with the electronic structure of the Weyl semimetal (four WPs and two Fermi arcs connecting them). This finding suggests a situation where the Fermi arcs' existence does not necessarily facilitate a Weyl orbit that is described in $\mathrm{Cd}_{3} \mathrm{As}_{2}[18,35]$, suggesting an unexplored scenario with various configurations of Fermi arcs.

\section{CONCLUSION}

In conclusion, we have demonstrated the band structure of the type-II TWS in $\mathrm{NbIrTe}_{4}$ with ARPES technique and found a good agreement with our ab initio calculation. We have shown that the $\mathrm{NbIrTe}_{4}$ has two different terminations with each its own rich SS feature and Fermi arc features connecting different pairs of WPs. Furthermore, the detailed analysis of the calculation result suggests that the SA on one surface rewires at different binding energies and creates a different intersurface Fermi arc loop (closed to open) which is attributed to the hybridization with the bulk band, calling for further research on different Fermi arc configurations. Our work may open insight on how the exotic Fermi arc evolves at different terminations within the same material and provide understanding for possible future applications.

\section{ACKNOWLEDGMENTS}

S.A.E. acknowledges the support from the Indonesian Endowment Fund for Education (LPDP) Scholarship. Y.L.C. acknowledges the support of the EPSRC Platform Grant (Grant No. EP/M020517/1) and CAS-Shanghai Science Research Center, Grant No. CAS-SSRC-YH-2015-01). C.F. acknowledges the financial support by the ERC Advanced Grant (No. 291472 Idea Heusler). J.J. acknowledges the support of the NRF, Korea through the SRC center for Topological Matter (No. 2011-0030787). C.Z thanks the financial support from the National Natural Science Foundation of China (Grant No. 11774427). Y.W.L acknowledges the support of the China Scholarship Council-University of Oxford Scholarship.
[1] A. Bansil, H. Lin, and T. Das, Rev. Mod. Phys. 88, 021004 (2016).

[2] N. P. Armitage, E. J. Mele, and A. Vishwanath, Rev. Mod. Phys. 90, 015001 (2018).

[3] B. H. Yan and C. Felser, Annu. Rev. Condens. Matter Phys. 8, 337 (2017).

[4] K. Manna, Y. Sun, L. Muechler, J. Kubler, and C. Felser, Nat. Rev. Mater. 3, 244 (2018).

[5] J. Jiang, Z. K. Liu, Y. Sun, H. F. Yang, C. R. Rajamathi, Y. P. Qi, L. X. Yang, C. Chen, H. Peng, C. C. Hwang, S. Z. Sun, S. K. Mo, I. Vobornik, J. Fujii, S. S. P. Parkin, C. Felser, B. H. Yan, and Y. L. Chen, Nat. Commun. 8, 13973 (2017).

[6] A. A. Soluyanov, D. Gresch, Z. J. Wang, Q. S. Wu, M. Troyer, X. Dai, and B. A. Bernevig, Nature 527, 495 (2015).

[7] Y. W. Li, Y. Y. Y. Xia, S. A. Ekahana, N. Kumar, J. Jiang, L. X. Yang, C. Chen, C. X. Liu, B. H. Yan, C. Felser, G.
Li, Z. K. Liu, and Y. L. Chen, Phys. Rev. Mater. 1, 074202 (2017).

[8] F. C. Chen, H. Y. Lv, X. Luo, W. J. Lu, Q. L. Pei, G. T. Lin, Y. Y. Han, X. B. Zhu, W. H. Song, and Y. P. Sun, Phys. Rev. B 94, 235154 (2016).

[9] S. Thirupathaiah, R. Jha, B. Pal, J. S. Matias, P. K. Das, P. K. Sivakumar, I. Vobornik, N. C. Plumb, M. Shi, R. A. Ribeiro, and D. D. Sarma, Phys. Rev. B 95, 241105(R) (2017).

[10] S. H. Khim, K. Koepernik, D. V. Efremov, J. Klotz, T. Forster, J. Wosnitza, M. I. Sturza, S. Wurmehl, C. Hess, J. van den Brink, and B. Buchner, Phys. Rev. B 94, 165145 (2016).

[11] Z. K. Liu, L. X. Yang, Y. Sun, T. Zhang, H. Peng, H. F. Yang, C. Chen, Y. Zhang, Y. F. Guo, D. Prabhakaran, M. Schmidt, Z. Hussain, S. K. Mo, C. Felser, B. Yan, and Y. L. Chen, Nat. Mater. 15, 27 (2016). 
[12] L. X. Yang, Z. K. Liu, Y. Sun, H. Peng, H. F. Yang, T. Zhang, B. Zhou, Y. Zhang, Y. F. Guo, M. Rahn, D. Prabhakaran, Z. Hussain, S. K. Mo, C. Felser, B. Yan, and Y. L. Chen, Nat. Phys. 11, 9 (2015).

[13] E. V. Gorbar, V. A. Miransky, I. A. Shovkovy, and P. O. Sukhachov, Phys. Rev. B 93, 235127 (2016).

[14] P. Roushan, J. Seo, C. V. Parker, Y. S. Hor, D. Hsieh, D. Qian, A. Richardella, M. Z. Hasan, R. J. Cava, and A. Yazdani, Nature 460, 1106 (2009).

[15] A. C. Potter, I. Kimchi, and A. Vishwanath, Nat. Commun. 5, 5161 (2014).

[16] D. Bulmash and X.-L. Qi, Phys. Rev. B 93, 081103(R) (2016).

[17] Y. Zhang, D. Bulmash, P. Hosur, A. C. Potter, and A. Vishwanath, Sci. Rep. 6, 23741 (2016).

[18] P. J. W. Moll, N. L. Nair, T. Helm, A. C. Potter, I. Kimchi, A. Vishwanath, and J. G. Analytis, Nature 535, 266 (2016).

[19] S. A. Parameswaran, T. Grover, D. A. Abanin, D. A. Pesin, and A. Vishwanath, Phys. Rev. X 4, 031035 (2014).

[20] H. Ishizuka, T. Hayata, M. Ueda, and N. Nagaosa, Phys. Rev. Lett. 117, 216601 (2016).

[21] B. Rosenstein, H. C. Kao, and M. Lewkowicz, Phys. Rev. B 95, 085148 (2017).

[22] Y. Baum, E. Berg, S. A. Parameswaran, and A. Stern, Phys. Rev. X 5, 041046 (2015).

[23] F. D. M. Haldane, arXiv:1401.0529.

[24] C. M. Wang, H. P. Sun, H. Z. Lu, and X. C. Xie, Phys. Rev. Lett. 119, 136806 (2017).

[25] A. Mar and J. A. Ibers, J. Solid State Chem. 97, 366 (1992).

[26] See Supplemental Material at http://link.aps.org/supplemental/ 10.1103/PhysRevB.102.085126 for detailed bulk calculations.

[27] J. Liu, H. Wang, C. Fang, L. Fu, and X. F. Qian, Nano Lett. 17, 1 (2017).
[28] L. Li, H. H. Xie, J. S. Zhao, X. X. Liu, J. B. Deng, X. R. Hu, and X. M. Tao, Phys. Rev. B 96, 024106 (2017).

[29] See Supplemental Material at http://link.aps.org/supplemental/ 10.1103/PhysRevB.102.085126 for the discussion on the band inversion and the topological $\mathrm{Z}_{2}$ invariant calculation.

[30] See Supplemental Material at http://link.aps.org/supplemental/ 10.1103/PhysRevB.102.085126 for detailed bulk calculations.

[31] K. Koepernik, D. Kasinathan, D. V. Efremov, S. Khim, S. Borisenko, B. Büchner, and J. v. d. Brink, Phys. Rev. B 93, 201101(R) (2016).

[32] I. Belopolski, P. Yu, D. S. Sanchez, Y. Ishida, T. R. Chang, S. S. Zhang, S. Y. Xu, H. Zheng, G. Q. Chang, G. Bian, H. T. Jeng, T. Kondo, H. Lin, Z. Liu, S. Shin, and M. Z. Hasan, Nat. Commun. 8, 942 (2017).

[33] E. Haubold, K. Koepernik, D. Efremov, S. Khim, A. Fedorov, Y. Kushnirenko, J. van den Brink, S. Wurmehl, B. Buchner, T. K. Kim, M. Hoesch, K. Sumida, K. Taguchi, T. Yoshikawa, A. Kimura, T. Okuda, and S. V. Borisenko, Phys. Rev. B 95, 241108(R) (2017).

[34] See Supplemental Material at http://link.aps.org/supplemental/ 10.1103/PhysRevB.102.085126 for detailed calculations.

[35] C. Zhang, A. Narayan, S. Lu, J. Zhang, H. Zhang, Z. Ni, X. Yuan, Y. Liu, J. H. Park, E. Zhang, W. Wang, S. Liu, L. Cheng, L. Pi, Z. Sheng, S. Sanvito, and F. Xiu, Nat. Commun. 8, 1272 (2017).

[36] H. M. Weng, C. Fang, Z. Fang, B. A. Bernevig, and X. Dai, Phys. Rev. X 5, 011029 (2015).

[37] J. W. Liu, C. Fang, and L. Fu, arXiv:1604.03947.

[38] H. F. Yang, L. X. Yang, Z. K. Liu, Y. Sun, C. Chen, H. Peng, M. Schmidt, D. Prabhakaran, B. A. Bernevig, C. Felser, B. H. Yan, and Y. L. Chen, Nat. Commun. 10, 3478 (2019). 